\title{
A practical approach to perioperative management of cardiac implantable electronic devices
}

Pow-Li Chia ${ }^{1}$, MBBS, MRCP, David $\underline{\text { FoO }}^{1}$, MBBS, MRCP

\begin{abstract}
With the increased use of cardiac implantable electronic devices (CIEDs), it is increasingly important to recognise the unique challenges involved in the management of patients with CIEDs who are undergoing surgery. Practice advisories and consensus statements have been issued by the American Society of Anesthesiologists and the Heart Rhythm Society, advocating a multidisciplinary approach. This review discusses and presents a practical approach to perioperative CIED management in the Singapore context.
\end{abstract}

Keywords: cardiac implantable electronic device, implantable cardioverter defibrillator, pacemaker

\section{INTRODUCTION}

Permanent cardiac pacemakers and implantable cardioverter defibrillators (ICDs) can be collectively referred to as cardiac implantable electronic devices (CIEDs). The number of CIED implantations worldwide, including in Singapore, has risen steadily over the years. ${ }^{(1)}$ As more people undergo CIED implantations, the likelihood of encountering such individuals in operative settings will increase. It is therefore important to be aware of the challenges involved in the safe management of these patients and their devices before, during and after operations.

In 2011, the American Society of Anesthesiologists published a practice advisory on the perioperative management of CIEDs. ${ }^{(2)} \mathrm{A}$ joint expert consensus statement on the same topic was subsequently promulgated by the American Society of Anesthesiologists and the Heart Rhythm Society, which recognised the importance of adopting a multidisciplinary approach. ${ }^{(3)}$ This review provides a broad overview of CIEDs and a safe practical approach to perioperative CIED management in the Singapore setting.

\section{Overview of CIEDs}

A permanent cardiac pacemaker is an implantable electronic device that treats significant, symptomatic bradyarrhythmia. It consists of a battery and electric circuitry housed within a pulse generator and transmits electrical impulses over leads that are in contact with the myocardium. A single chamber pacemaker consists of a pulse generator with a single lead placed in the right ventricle. A dual chamber pacemaker has one lead in the right ventricle and another in the right atrium. Biventricular pacemakers typically have three leads implanted in the right ventricle, right atrium and the coronary sinus, respectively, which allow pacing of the left ventricle. A pacemaker with additional features to treat ventricular tachyarrhythmias by way of defibrillation and antitachycardia pacing is termed an ICD. ICDs may be implanted for primary or secondary prevention of sudden cardiac death from ventricular tachyarrhythmia.
The programming modes of CIEDs are described using the generic code of the North American Society of Pacing and Electrophysiology/British Pacing and Electrophysiology Group. ${ }^{(4)}$ The code consists mainly of three letters, which denote the chamber being paced, chamber where the sensing of intrinsic cardiac signals occurs and response to sensed intrinsic signals, respectively. AOO, VOO and DOO modes indicate asynchronous pacing, in which pacing stimuli is delivered regardless of sensed intrinsic signals. AAI, VVI, DDD, DDI are modes that pace the heart only in the absence of sensed inherent cardiac signals at preprogrammed settings. Certain CIEDs have a feature known as rate modulation. Through the use of sensors such as accelerometers to detect movement or monitors to track changes in thoracic impedance, these CIEDs pace the heart at a faster rate when they detect that the patients are active as opposed to sedentary. Rate modulation helps to alleviate symptoms associated with chronotropic incompetence.

All patients with CIEDs are given a CIED information card which states the manufacturer, model and serial numbers of the pulse generator and leads, the date of implantation and the contact numbers of the respective industry employed allied health professionals (IEAP) in the event of an emergency. It is important to identify the CIED manufacturer, as each of the four major CIED vendors in Singapore (Medtronic, St Jude Medical, Boston Scientific and Biotronik) have unique programmers for device interrogation and programming. In the absence of the CIED information card, chest radiography can be useful for device identification. Jacob et al described an algorithm for CIED identification using radiography in 2011. ${ }^{(5)}$ At the very least, chest radiography allows physicians to differentiate pacemakers from ICDs and to evaluate the number and position of leads. Fig. 1 illustrates a dual chamber pacemaker. The device in Fig. 2, however, is identified as an ICD: the thick radio-opaque segments in the right ventricular lead are the high voltage shocking coils. A CIED with a coronary sinus lead, as depicted in Fig. 3, can be recognised as a biventricular or cardiac resynchronisation therapy device. 


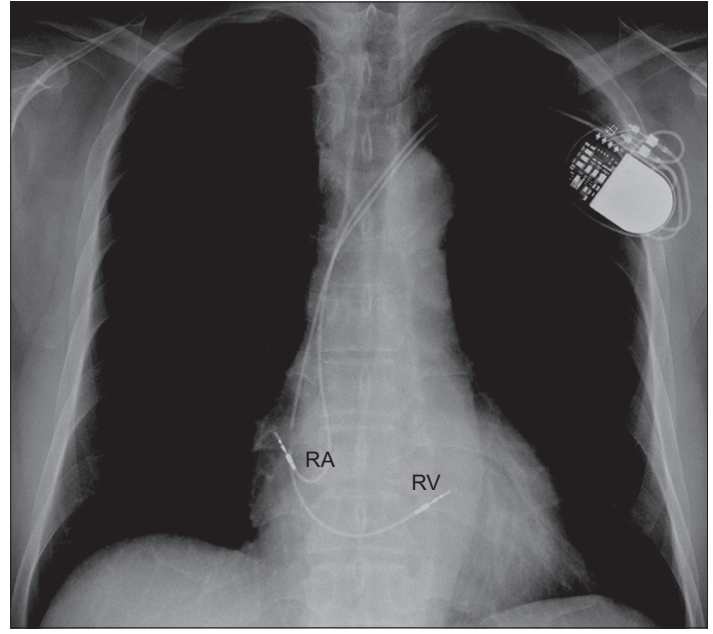

Fig. 1 Chest radiograph shows a dual chamber pacemaker with one lead implanted in the right ventricle (RV) and another in the right atrium (RA).

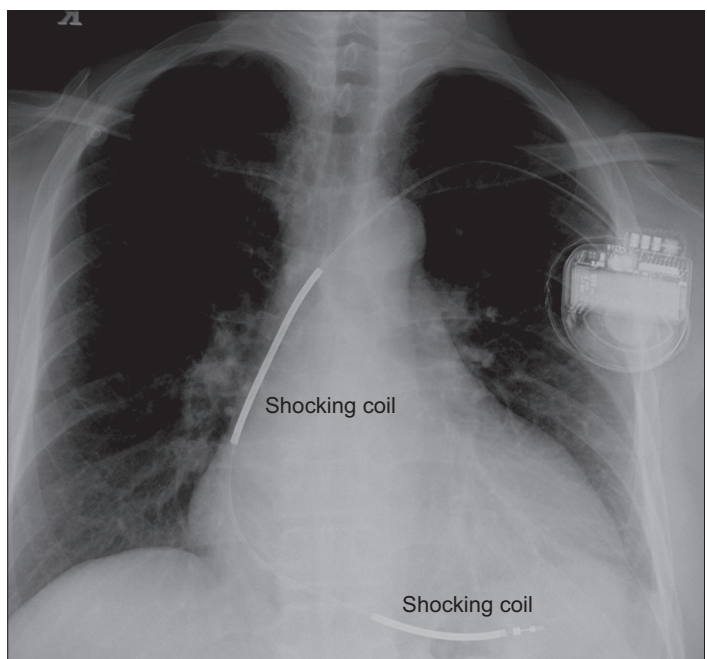

Fig. 2 Chest radiograph shows two radio-opaque thick segments along the right ventricular lead that are the high voltage shocking coils, identifying the device as a single chamber implantable cardioverter defibrillator.

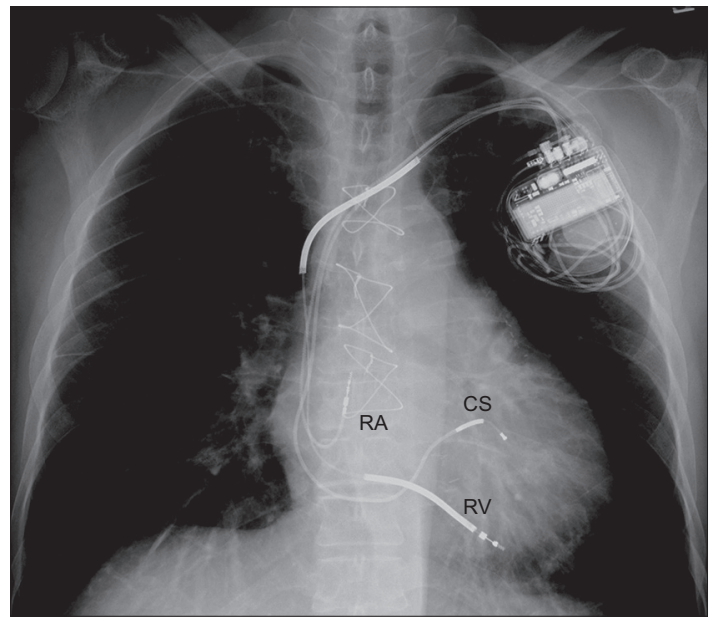

Fig. 3 Chest radiograph shows a device with a right ventricular (RV) lead, a right atrial (RA) lead, a coronary sinus (CS) lead and radio-opaque thick segments along the RV lead. These features identify the device as a biventricular implantable cardioverter defibrillator, also referred to as a cardiac resynchronisation therapy defibrillator.

\section{PERIOPERATIVE MANAGEMENT OF CIEDS}

\section{Preoperative period}

It is imperative that the proceduralists and anaesthetists recognise the presence of CIEDs in patients prior to the operation. The type of CIED can be identified through history-taking, patient medical records, CIED information cards and chest radiography. Following identification of the CIED, it is useful to involve the cardiologist, as a multidisciplinary approach to perioperative CIED management ensures better outcomes. ${ }^{(3)}$ The CIEDs should ideally have been checked within the last six months to determine the pacing burden, hence ensuring optimal lead parameters and device function. ${ }^{(2,3)}$ Patients who have a pacing burden of $\geq 40 \%$ are deemed to be pacing dependent. In the absence of CIED interrogation records, an electrocardiogram that reveals a predominately paced rhythm also implies pacing dependence.

In patients with CIEDs who are undergoing operations, the main concern is the possible effects of electromagnetic interference (EMI) on device function. Possible sources of EMI include electrocautery (especially monopolar electrocautery), evoked potential monitors, nerve stimulators, external defibrillation, radiofrequency ablation and extracorporeal shock wave lithotripsy. For pacemakers, EMI may be misinterpreted as intrinsic cardiac signals, resulting in oversensing and inhibition of pacing. In patients who are pacing dependent, inappropriate inhibition of pacing due to EMI can cause significant bradycardia, sinus arrest or ventricular standstill. In ICDs, EMI can cause noise, which provokes inappropriate defibrillatory shocks. EMI may also potentially result in failure of ICD therapy during ventricular tachyarrhythmias.

Several measures may be taken to minimise EMI effects on CIEDs. Potential sources of EMI should be placed at least $15 \mathrm{~cm}$ away from the CIED pulse generator. ${ }^{(2)}$ The use of bipolar electrocautery is recommended. If monopolar electrocautery is required, short, intermittent, irregular bursts of diathermy at minimal power settings, using 'cut' instead of 'coagulation' current, will reduce EMI. Cautery dispersal pads should be placed in a position such that the current is directed away from the CIED pulse generator. ${ }^{(6)}$ Defibrillation/pacing pads should be placed on the chest in an anterior-posterior arrangement instead of directly over the pulse generator.

The decision on the need for CIED check and reprogramming before operating depends on the nature of the procedure (having identified possible EMI sources), type of CIED and the patient's pacing burden. CIED reprogramming is indicated in ICD patients, procedures with potential EMI and patients who are pacing dependent (i.e. pacing burden $\geq 40 \%$ ). For such cases, device reprogramming may entail switching off ICD antitachycardia therapies (i.e. antitachycardia pacing and defibrillation), converting the pacing mode to an asynchronous one (i.e. VOO, DOO) and/or deactivating rate modulation features. Patients who do not fulfil the listed criteria generally do not require preoperative CIED reprogramming and the procedure can proceed with a cardiac device magnet on standby. 


\section{Intraoperative and postoperative period}

Patients with CIEDs must be closely monitored, especially via electrocardiographic and haemodynamic monitoring, during the operation to ascertain changes in pacing behaviour. Defibrillation/pacing pads should be placed on the patients' chests in an anterior-posterior configuration so that, should CIED malfunction occur, backup pacing or external defibrillation can be administered.

The use of cardiac device magnets to alter device behaviour intraoperatively is convenient, reliable and does not require special training. These magnets have to be placed and secured directly over the CIED pulse generator to effect the changes. In pacemakers, magnets switch off any rate modulation features and convert them to asynchronous pacing modes, which prevent EMI-induced pacing inhibition. The paced heart rate during asynchronous pacing is dependent on device programming, battery longevity and manufacturer-defined defaults. Asynchronous pacing will continue until the magnet is removed from contact with the CIED, at which point the device will revert to its baseline programmed settings. Asynchronous pacing modes should not be used in nonpacing dependent patients as the pacing stimulus may trigger the 'R-on- $\mathrm{T}$ ' phenomenon, resulting in ventricular tachyarrhythmias. In ICDs, magnet application switches off arrhythmia detection and antitachycardia therapies, which reactivate once the magnet is removed. Magnet application does not affect the pacing mode of ICDs; hence, formal device reprogramming is needed if ICDs need to be switched to asynchronous pacing modes in patients who are pacing dependent. Device reprogramming should also be performed during surgeries in which patients are placed in a nonsupine position, as this position makes magnet application unstable. In individuals who are extremely obese, the magnetic field of cardiac device magnets may not reach the CIED and device reprogramming is advisable. ${ }^{(7)}$
After the operation, continued patient monitoring is necessary until CIED function is restored to baseline settings. Formal CIED interrogation to ensure optimal device function should be performed at the next available opportunity by the cardiology or IEAP team. Proposed algorithms for perioperative CIED management in different possible scenarios are shown in Figs. 4-6. For emergency procedures, there may not be sufficient time for preoperative CIED checks or identification. The routine application of cardiac device magnets over the CIED pulse generator will deactivate antitachycardia therapies if the

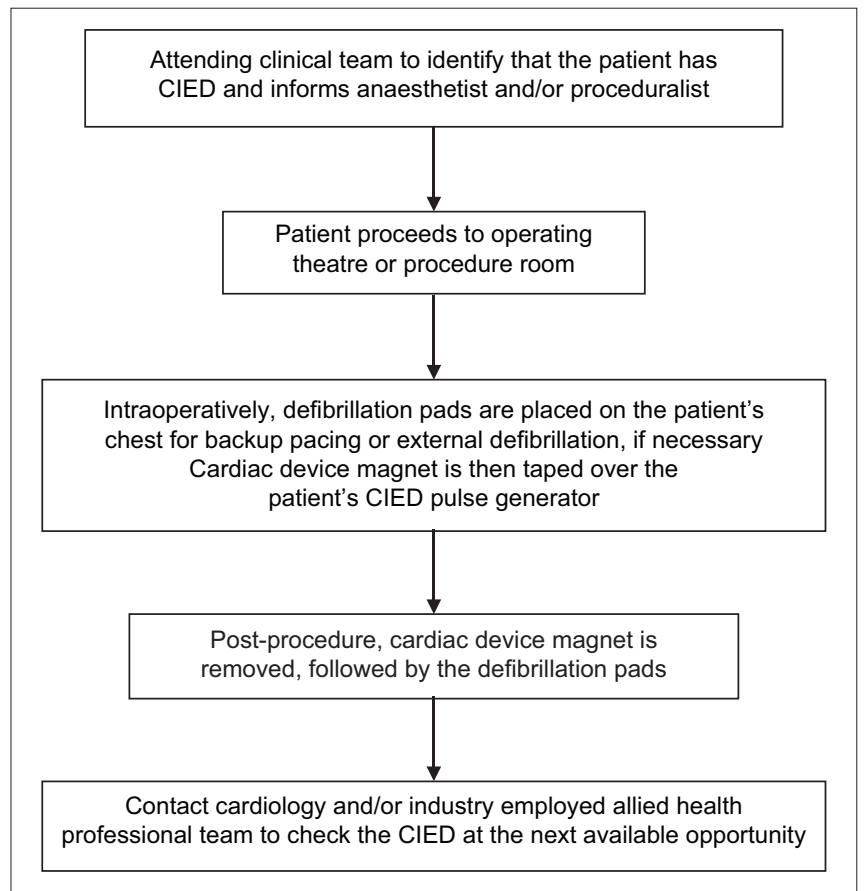

Fig. 4 Flowchart of proposed algorithm for the perioperative management of patients with cardiac implantable electronic devices (CIED) in an emergency setting.

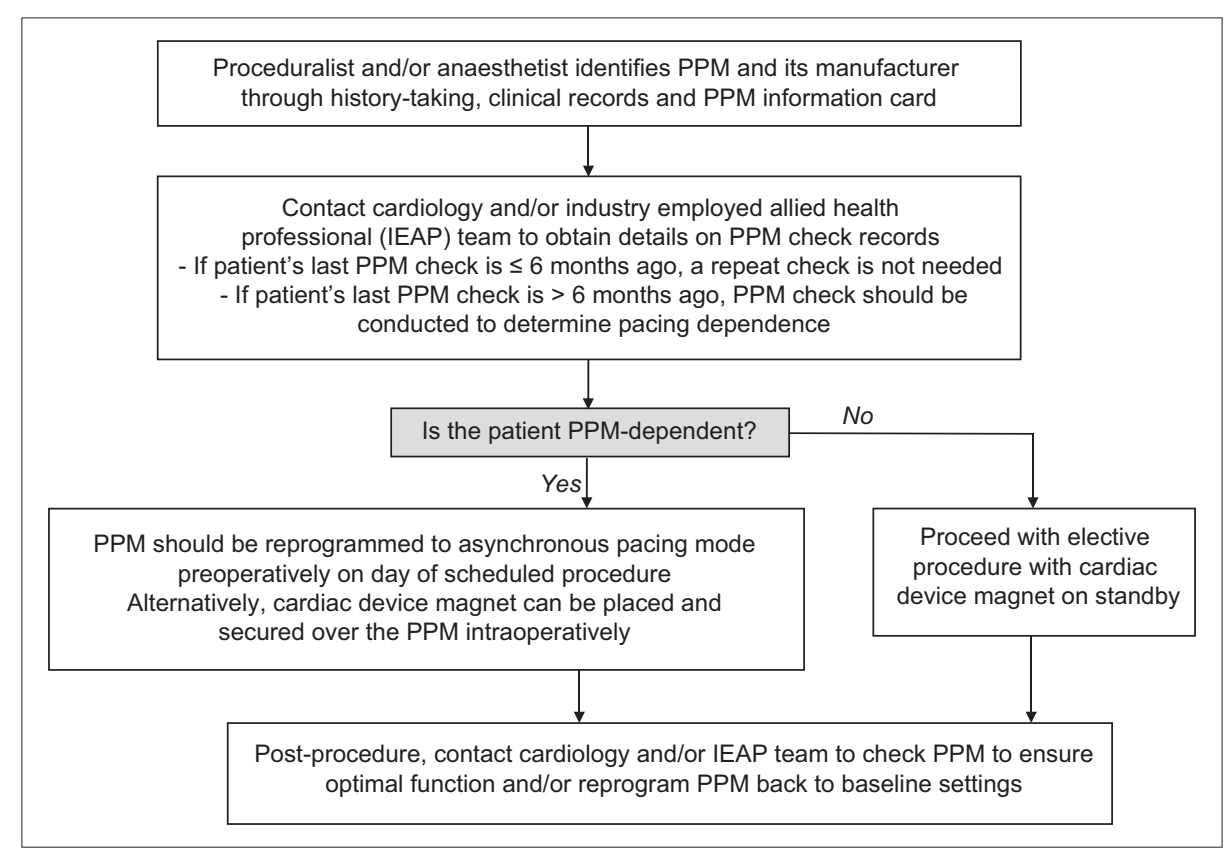

Fig. 5 Flowchart of proposed algorithm for the perioperative management of patients with cardiac permanent pacemakers (PPM) in an elective, non-emergency setting. 


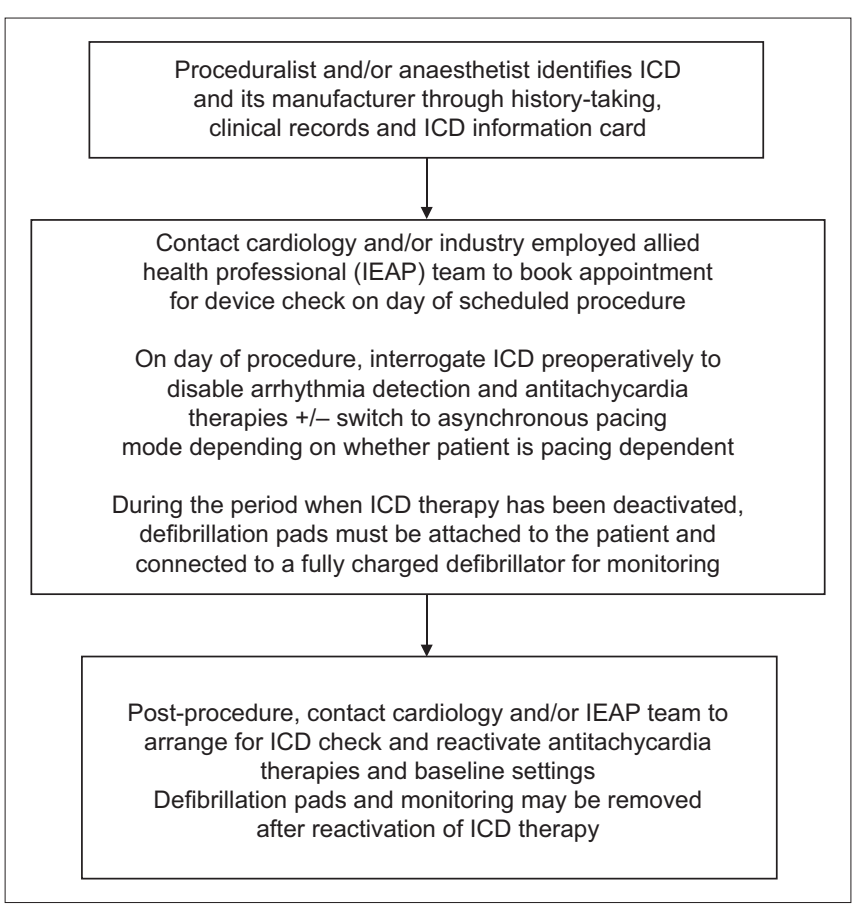

Fig. 6 Flowchart of proposed algorithm for the perioperative management of patients with implantable cardioverter defibrillators (ICD) in an elective, non-emergency setting.

device is an ICD or a pacemaker, as the magnet will switch it to the asynchronous pacing mode. Defibrillation/pacing pads should be applied to the chest intraoperatively in anticipation of possible CIED malfunction, in which case the pads can be used for backup pacing or external defibrillation. For non-emergency elective procedures, effort and time should be spent to identify the CIED, and appropriate checks and reprogramming performed if necessary.

\section{CONCLUSION}

Adopting a multidisciplinary approach that involves the proceduralist, anaesthetist, cardiologist and IAEP is ideal for safe perioperative CIED management. As cardiac device magnets can be reliably used intraoperatively to alter device behaviour, the urgency of the procedures does not need to be compromised by device reprogramming. The decision-making process should be tailored to individual patients and their needs, with the aim of preventing haemodynamic embarrassment consequent to CIED malfunction.

\section{REFERENCES}

1. Ruiz N, Buisán F, Fulquet E. [Implantable pacemakers and defibrillators: implications for anesthesia and perioperative management]. Rev Esp Anestesiol Reanim 2009; 56:97-107. Spanish.

2. American Society of Anesthesiologists. Practice advisory for the perioperative management of patients with cardiac implantable electronic devices: pacemakers and implantable cardioverter-defibrillators: an updated report by the american society of anesthesiologists task force on perioperative management of patients with cardiac implantable electronic devices. Anesthesiology 2011; 114:247-61.

3. Crossley GH, Poole JE, Rozner MA, et al. The Heart Rhythm Society (HRS)/American Society of Anesthesiologists (ASA) Expert Consensus Statement on the perioperative management of patients with implantable defibrillators, pacemakers and arrhythmia monitors: facilities and patient management: executive summary this document was developed as a joint project with the American Society of Anesthesiologists (ASA), and in collaboration with the American Heart Association (AHA), and the Society of Thoracic Surgeons (STS). Heart Rhythm 2011; 8:e1-18.

4. Bernstein AD, Daubert JC, Fletcher RD, et al. The revised NASPE/BPEG generic code for antibradycardia, adaptive-rate, and multisite pacing. North American Society of Pacing and Electrophysiology/British Pacing and Electrophysiology Group. Pacing Clin Electrophysiol 2002; 25:260-4.

5. Jacob S, Shahzad MA, Maheshwari R, Panaich SS, Aravindhakshan R. Cardiac rhythm device identification algorithm using X-Rays: CaRDIA-X. Heart Rhythm 2011; 8:915-22.

6. Fiek M, Dorwarth U, Durchlaub I, et al. Application of radiofrequency energy in surgical and interventional procedures: are there interactions with ICDs? Pacing Clin Electrophysiol 2004; 27:293-8.

7. Jacob S, Panaich SS, Maheshwari R, et al. Clinical applications of magnets on cardiac rhythm management devices. Europace 2011; 13:1222-30. 